\title{
Thymoquinone-loaded PLGA nanoparticles: antioxidant and anti-microbial properties
}

\author{
*Ilaiyaraja Nallamuthu1, Ambica Parthasarathi², Farhath Khanum ${ }^{1}$ \\ ${ }^{1}$ Biochemistry and Nanosciences Discipline, Defence Food Research Laboratory (DFRL), Siddharthanagar, Mysore-570011, India \\ ${ }^{2}$ Department of Microbiology, University of Mysore, Manasagangotri, mysore-570014, India
}

\begin{abstract}
The aim of the present study was to synthesize and characterize the Thymoquinone (TQ) encapsulated PLGA (poly (dl-lactide-coglycolide) nanoparticles, and further evaluate for its antioxidant and anti-bacterial activities. TQ is a potential active ingredient of Nigella sativa seed and possess a spectrum of therapeutic properties. Nanoparticles were prepared according to solid-in-oil-in-water (s/o/w) solvent evaporation method. Dynamic laser light scattering (DLS) and SEM studies indicated a mean particle size of $<200$ $\mathrm{nm}$. The success of encapsulation was confirmed by FTIR technique, and the encapsulation efficiency (EE) of TQ was determined to be $62 \%$. In vitro drug release study showed a maximum release of TQ at $75 \%$ and $54 \%$ respectively for artificial intestinal and gastric juices over the period of 7 days. DPPH radical scavenging activity of the nanoparticles was found to be $71 \%$ at $1 \mathrm{mg} / \mathrm{ml}$ concen tration. It also exhibited antibacterial property against E. coli, Staphylococcus aureus and Salmonella typhi strains, tested using well diffusion method. In conclusion, our study shows that PLGA encapsulated TQ nanoparticle with sustained release property has preserved antioxidant as well as anti-microbial activity, and therefore suggesting its therapeutic applications in various food samples.
\end{abstract}

Key Words: Encapsulation, particle size, in vitro release kinetics, thermal stability.

\section{INTRODUCTION}

Thymoquinone (TQ) is a major active constituent of Nigella sativa (black seeds). The seeds have been used in traditional medicines to treat a variety of ailments and most of its biological effects are mainly attributed to TQ. The content of TQ in seed is $2200 \mathrm{mg} / \mathrm{kg}$ on fresh weight basis. The therapeutic properties of TQ include antioxidant (Mansoor et al., 2002), anti-inflammatory (Umar et al., 2012), anti-diabetic (Pari and Sankaranarayanan, 2009) and hepato-protective (Abdel-Wahab, 2013), neuroprotective (Al-Majed et al., 2006; Alhebshi et al., 2013), anticancerous (Gali-Muhtasib et al., 2006; Woo et al., 2012) anti-ulcerative (Arslan et al., 2005; Magdy et al., 2012), antmicrobial (Harzallah et al., 2011), immunomodulatory (ElMahmoudy et al., 2002) properties etc.

There is a growing interest in use of phytochemical as nutraceutical agents in pharmaceutical and food formulations in the past few decades. Although, TQ has tremendous potential as a therapeutic compound but its effectiveness and oral bioavailability is limited by poor solubility and poor formulation characteristics of high lipophilicity. In the recent past, research works are being focused on improving poor bioavailable drugs and phytocompounds by nanoencapsulation technique. The nature of carrier material (chitosan, cyclodextrins, PLGA etc.) has significant effects on pharmacokinetics and pharmacodynamics of bioactive compounds. Poly (lacticco-glycolic acid) is a biocompatiable and biodegradable copolymer has been employed to increase oral bioavailability of several bioactives (Derakhshandeh et al., 2011; Ma et al., 2012; Srivastava et al., 2013). In body, it is degraded into nontoxic lactic acid and glycolic acid. Studies report

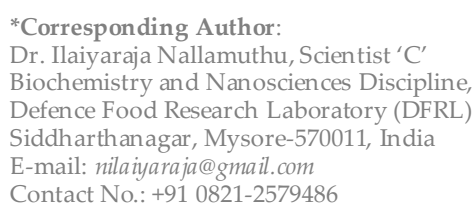

that PLGA is a non-toxic polymer based on cell culture and animal experiments (Semete et al., 2010).

In the present study, TQ loaded PLGA nanoparticle was prepared using PVA as a stabilising agent. The particles were characterised for particle size, morphology, encapsulation efficiency, in vitro release, antioxidant and anti-microbial activity.

\section{MATERIALS AND METHODS}

Polylactic acid/polyglycolic acid PLGA (50:50, MW: 500015,000), Poly (vinyl alcohol) (PVA, MW: 9000-10,000), sucrose, monosodium phosphate $\left(\mathrm{NaH}_{2} \mathrm{PO}_{4}\right)$, thymoquinone were purchased from sigma. All organic solvents were of HPLC grade, and other chemicals were of analytical grade. Milli-Q grade water was used for the preparation of solution and mobile phase.

\section{Preparation of PLGA-TQ nanoparticles}

Nanoparticles were prepared with poly (dl-lactide-coglycolide) using $\mathrm{S} / \mathrm{O} / \mathrm{W}$, an emulsification-solvent evaporation/diffusion method with slight modifications (Xie et al., 2011). Briefly, $45 \mathrm{mg}$ of PLGA was dissolved in $2 \mathrm{ml}$ of dicholoromethane (HPLC-grade) as an oil phase for $12 \mathrm{hr}$ in at room temperature to obtain uniform solution. $5 \mathrm{mg}$ of bioactive liphophillic compound-TQ was added to the above solution. Then the suspension was sonicated for 2 minutes to generate S/O primary emulsion. This mixture was emulsified with an aqueous phase of 20 $\mathrm{ml}$ of PVA $(1 \% \mathrm{w} / \mathrm{v})$ to form $\mathrm{S}-\mathrm{O} / \mathrm{W}$ emulsion by rotating in Magnetic stirrer (REMI, India) at $400 \mathrm{rpm}$. Once all the compound/polymer mixture was added the contents were vortexed for $10 \mathrm{sec}$ at a high setting. The resulting suspension was sonicated (Ultra Sonicator bath- INKARP) for $3 \mathrm{~min}$ to generate the final $\mathrm{S} / \mathrm{O} / \mathrm{W}$ emulsion. Then organic solvent present in suspension was evaporated by rotary vacuum evaporation using Rotary evaporator (Heidolph) at $50^{\circ} \mathrm{C}$. The nanoparticles were then collected by centrifugation at $10000 \mathrm{~g}$ for $20 \mathrm{~min}$ at $4^{\circ} \mathrm{C}$. Finally, they 
were resuspended in $2 \mathrm{ml}$ of cryoprotectent solution (2\% sucrose), dried on a Lyophilizer (Lyophilisation Systems India Ltd) and stored at $4^{\circ} \mathrm{C}$.

\section{Particle size and Zeta potential}

Particle size and size distribution were measured by dynamic laser scattering technique using Malvern Zetasizer (Model no: nano-ZS90, Malvern Instruments, UK). Nanoparticles, $10 \mathrm{mg} / \mathrm{ml}$ were suspen ded in distilled water, then vortexed and sonicated for a few minutes. Each sample was measured in triplicate. The particle size distribution of the nanoparticles is reported as a polydispersity index (PDI), a measure of the distribution broadness of the particle size. $2 \mathrm{ml}$ of sample was taken in cuvette and analyzed at $25^{\circ} \mathrm{C}$ with an angle of $90^{\circ}$.

\section{Scanning Electron Microscopy (SEM)}

The morphology of the nanoparticle was determined by scanning electron microscope $(\mathrm{S} 3700 \mathrm{~N}$, Hitachi) at an accelerating voltage of $15.0 \mathrm{Kv}$. One drop of nanoparticles was placed on a graphite surface and after the sample had dried it was coated with gold using ion sputter.

\section{FT-IR spectroscopic study}

FTIR spectroscopy measurements were carried out to recognize the bio-groups that bound distinctively. During FTIR analvsis, a spot on the specimen is subjected to a modulated IR beam. The specimen's transmittance and reflectance of the infrared rays at different frequencies is translated into an IR absorption plot consisting of reverse peaks. TQ, PLGA, PVA, TQ nanoparticles were mixed with the $\mathrm{KBr}$ to result in pellet and then examined for FTIR spectra with the set range of 400 to $4000 \mathrm{~cm}^{-1}$ (Frontier Optica, PERKIN ELMER).

\section{Encapsulation efficiency}

Nanoparticle solution was centrifuged at $30,000 \mathrm{~g}$ for $15 \mathrm{~min}$. After centrifugation, the supernatant was removed and $1 \mathrm{ml}$ of methanol was added to the pellets and then treated with sonication for $5 \mathrm{~min}$ to release TQ from the particles. The amount of TQ was measured by HPLC method. The isocratic mobile phase consisted of water: methanol: 2-propanol (50:45:5) and run at the flow rate of $0.8 \mathrm{ml} / \mathrm{min}$. UV detection of TQ was carried out at the wave length of $254 \mathrm{~nm}$ (Ghosheh et al., 1999).

Encapsulation efficiency $(\%)=\frac{\text { weight of CGA in nanoparticle }}{\text { weight of total CGA }} \times 100$

\section{In-vitro release kinetics}

$100 \mathrm{mg}$ of nanoparticles separated by centrifugation at $30,000 \mathrm{~g}$ and the pellet was dissolved in $2 \mathrm{ml}$ of phosphate buffer saline (PBS pH 7.4) and $0.1 \mathrm{M} \mathrm{HCl}$ and transferred to a dialysis membrane with a molecular cut off range of 7 KDa (Cat no: 68700, Pierce Make). The bag was suspended in $100 \mathrm{ml}$ of phosphate buffer under magnetic stirring condition for 7 days (Tsai et al., 2011). $1 \mathrm{ml}$ of the released sample was removed at regular interval of time, and the amount of TQ in the release medium was evaluated by HPLC method.

\section{Effect of temperature on nanoparticles}

$3 \mathrm{ml}$ of TQ-PLGA nanoparticles solution was taken in tubes and heated for 5,10 and 15 minutes at $60^{\circ} \mathrm{C}, 80^{\circ} \mathrm{C}$ and $100^{\circ} \mathrm{C}$ respectively. The change in the physicochemical properties of the nanoparticles after heat treatment was measured in terms of particle size and zeta potential (Jang and Lee, 2008).

\section{In-vitro Antioxidant capacity of encapsulated TQ}

$\mathrm{DPPH}$ radical scavenging activity of nanoparticle was determined according to the method of Blois (Braca et al. 2001). Briefly, $0.1 \mathrm{~mL}$ of different concentration of nanoparticles $(0.1-1 \mathrm{mg} / \mathrm{ml})$ was added to $3 \mathrm{~mL}$ of DPPH $(1$, 1-diphenyl-2-picrylhydrazyl) $(0.004 \% \mathrm{DPPH})$ solution $(0.2$ $\mathrm{mM}$ in methanol) as the free radical source. The mixture was shaken and kept for 45 minutes at room temperature. The decrease of solution absorbance due to proton donating activity of components of each nanoparticle was determined at $517 \mathrm{~nm}$. Lower absorbance of the reaction mixture indicated higher free radical scavenging activity. Vitamin C (L-ascorbic acid) was used as the positive control. The DPPH radical scavenging activity was calculated using the following formula:

$D P P H$ radical scavenging activity $(\%)=\left[\frac{A^{0}-A 1}{A^{0}}\right] \times 100$

Where, $\mathrm{A}^{\circ}$ is the absorbance of the control, and $\mathrm{A} 1$ is the absorbance of extract or standard sample.

Antibacterial property by well diffusion method

Antibacterial property test were performed by a modified agar-well diffusion method (Okeke et al., 2001). A $0.1 \mathrm{ml}$ volume of the standard suspension of each test bacterial strain was spread evenly on Nutrient Agar using a sterile glass rod spreader and the plates were allowed to dry at room temperature. Subsequently, 6-mm diameter wells were bored in the agar and a $250 \mu \mathrm{l}$ volume of $0.25 \mathrm{mg} / \mathrm{ml}$ of nanoparticle were transferred into the wells. After holding the plates at room temperature for $2 \mathrm{~h}$, they were incubated at $37^{\circ} \mathrm{C}$ for $24 \mathrm{hr}$. Inhibition zone diameter (IZD) was measured to the nearest millimeter ( $\mathrm{mm}$ ).

\section{RESULTS AND DISCUSSION}

Encapsulated bioactive compounds into nanometric delivery systems are being increasingly tested in food system with the intention to improve the bioavailability of the hydrophobic phytocompounds. Selection of right kind of polymer becomes essential to minimize the impact on the quality attributes of the final product. At present, PLGA is extensively used in drug delivery systems. There are several reports published in the recent years on the use of PLGA polymer as an ideal carrier system for the encapsulation of substances like curcumin (Yallapu et al., 2010), ferulic acid (Merlin et al., 2012), quercitrin (Kumari et al., 2011), vitamin E (Chaiyasat et al., 2013, saponin (Ven et al., 2011), and plant extracts (Samadder et al., 2012; Das et al., 2012) etc. PLGA has the property to cross the bloodbrain barrier and therefore it is a suitable polymer for compounds used in treating neurological and psychological disorders as well.

In the present study, Thymoquinone encapsulated PLGA Nanoparticles were prepared by a standard method of solvent evaporation technique using PVA as a stabilizer. PLGA is one of the widely used delivery system for the controlled release of hydrophobic substances.

\section{Physiochemical properties of nanoparticles}

Size and zeta potential

Physicochemical properties such as size, morphology, charge, and physical state are the critical factors that influence the functional performance of any nanoparticle based delivery systems (Ahsan et al., 2002). We therefore 
Size Distr bution by Intensity

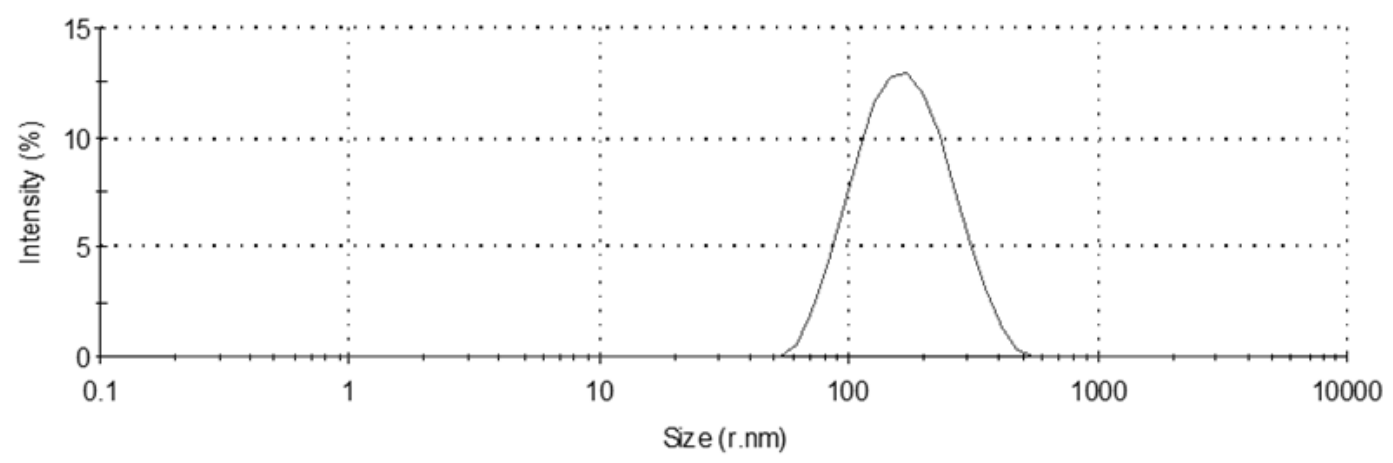

Record 41: TQ NANO LY OPHIUZED 14/3/132

Figure 1: Particle size distribution of TQ loaded PLGA nanoparticles.

Zeta Potential Distribution

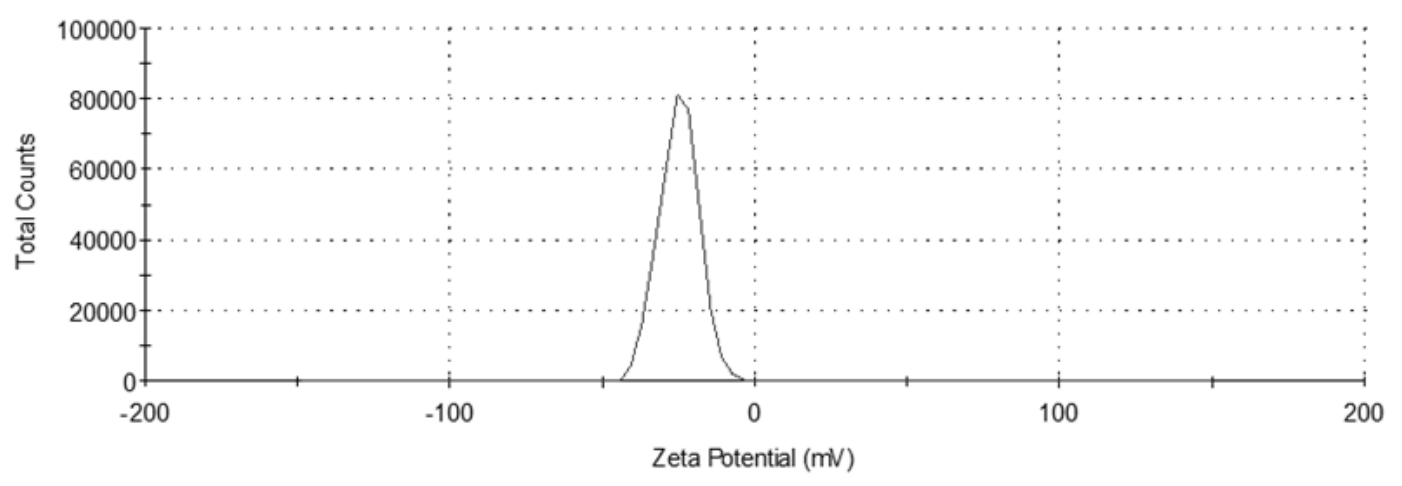

Record 43: TQ NANO LYOPHUZED14/3/13 1

Figure 2: Zeta potential distribution of TQ loaded PLGA nanoparticles.
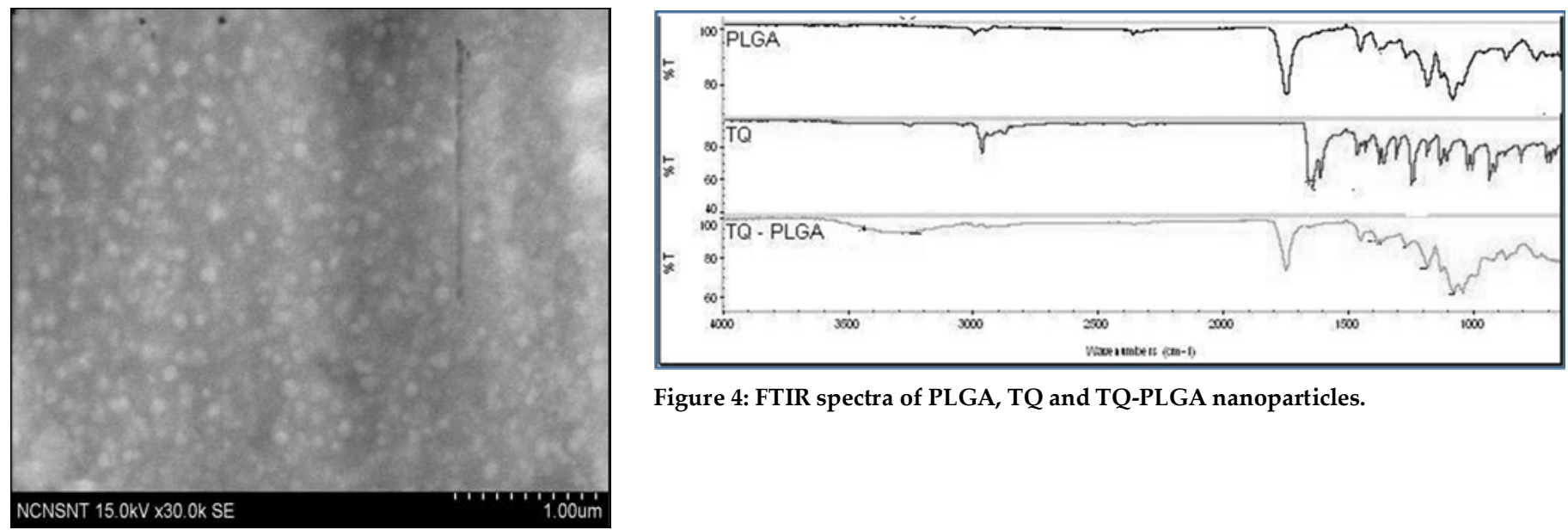

Figure 4: FTIR spectra of PLGA, TQ and TQ-PLGA nanoparticles.

Figure 3: SEM image of TQ loaded PLGA nanoparticles. 


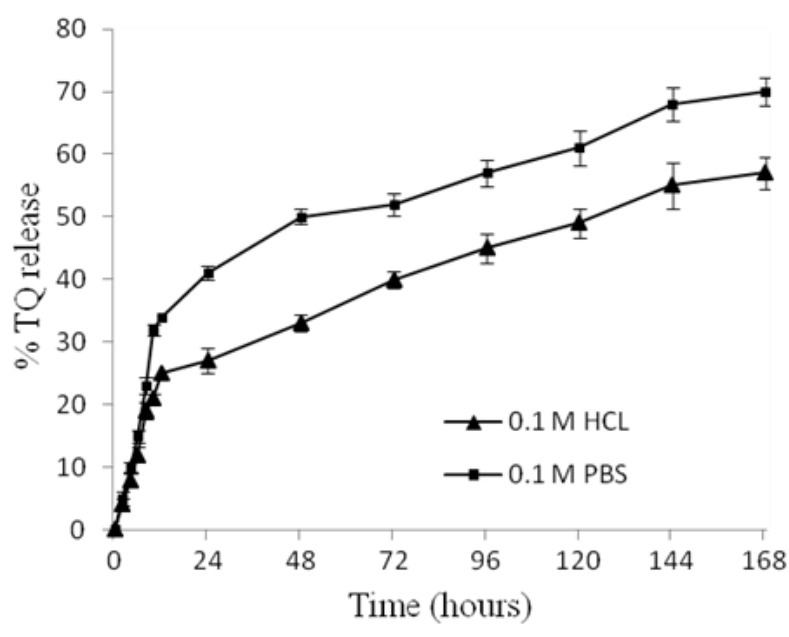

Figure 5: In vitro release kinetics of TQ from nanoparticle for the period of 7 days.

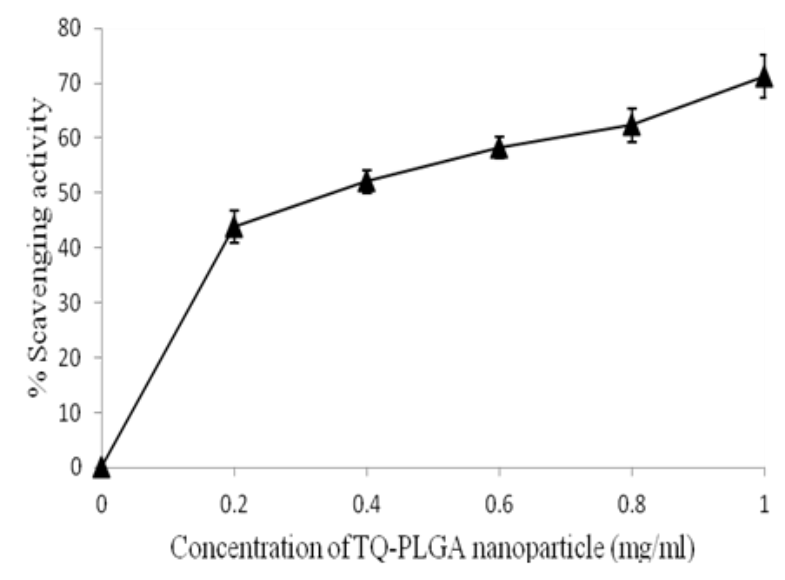

Figure 6: DPPH radical scavenging activity of TQ loaded PLGA nanoparticles.

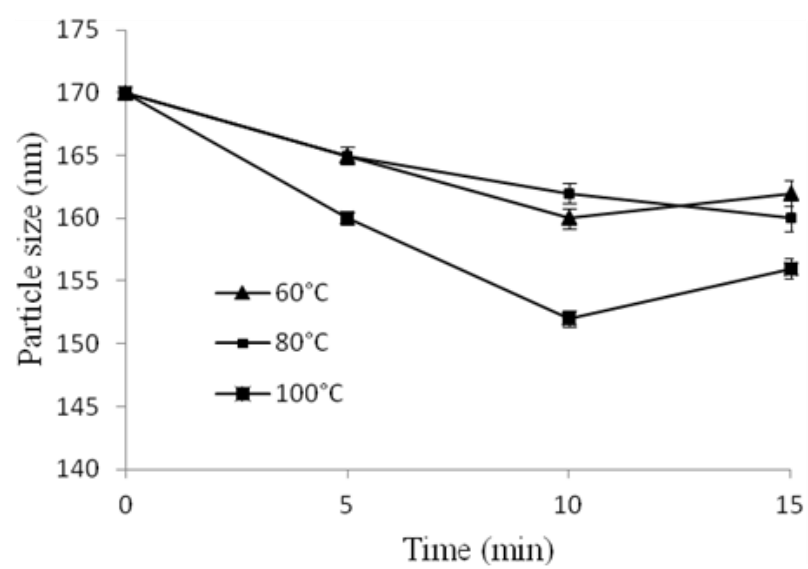

(a)

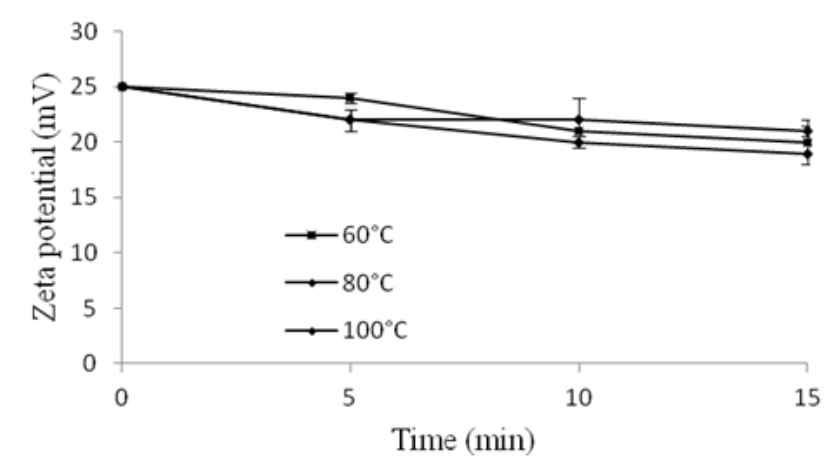

(b)

Figure 7: Effect of heat treatment at 60,80 and $100^{\circ} \mathrm{C}$ on: (a) particle size and (b) Zeta potential of TQ loaded PLGA nanoparticles.
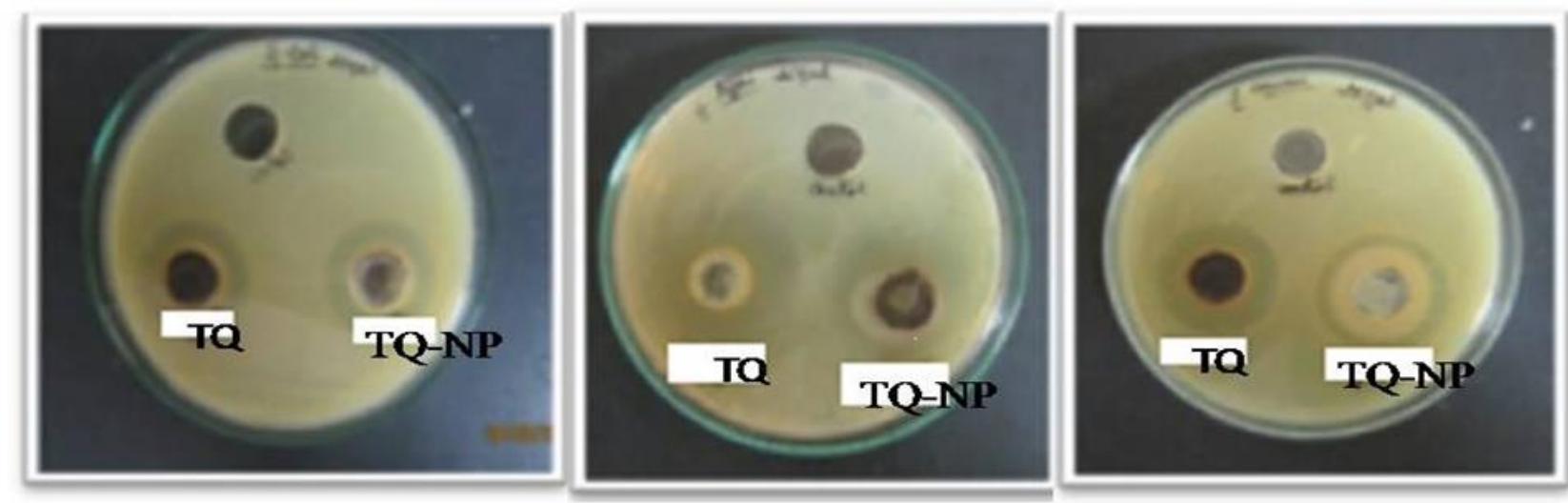

Figure 8: Anti-microbial properties of TQ loaded PLGA nanoparticles against E. coli, Staphylococcus aureus and Salmonella typhi. 
measured the particle size distribution pattern of TQ nanoparticle (figure 1). From the results, the calculated average particle size of TQ nanoparticle was $148 \mathrm{~nm}$ with the narrow distribution of polydispersity index (PDI) i.e. 0.2 . The average zeta potential value was $-24.8 \mathrm{mV}$ (figure $2)$, suggesting higher stability of nanoparticles. In general, zetapotential of more than $+30 \mathrm{mV}$ or less than $-30 \mathrm{mV}$ is considered as a standard value in providing enough repulsion forces to avoid particle aggregation (Qi et al., 2004). These results agree with the published reports by others.

Bhattacharyya et al. (2010) have reported recently that PLGA encapsulated ethanolic extract of plant sample (Gelsemium sempervirenst) was at $122 \mathrm{~nm}$ and zeta potential was $14.8 \mathrm{mV}$.

\section{SEM study}

The structure of the nanoparticles plays an important role in determining their adhesion to and interaction and absorption with the body cells. To determine the morphology of the nanoparticles formed, SEM was carried out. The particles appeared as spherical shape with smooth surface and the size of the particles was found to be $<200 \mathrm{~nm}$ (figure 3). Recent studies have demonstrated that the molecular weight of PLGA, organic phase and PVA concentration attributes to the variation in the size of the nanoparticle prepared. Nanoparticles with smaller size can lead to improvement in bioavailability because they would have greater ease of entry and durability in the cells. Savic et al. (2003) have reported that the large particles of less than $5 \mathrm{~mm}$ would be taken up via the lym phatics whereas smaller particles of less than $500 \mathrm{~nm}$ can cross the membrane of epithelial cells through endocytosis.

\section{Characterization of nanoparticles \\ FTIR}

FTIR measurement was carried out to to detect the functional groups of compounds. It is based on the fact that bonds and groups of bonds vibrate at characteristic frequencies. A molecule that is exposed to infrared rays absorbs infrared energy at frequencies which are characteristic to that molecule. The infrared spectra of PLGA, PVA TQ are shown in figure 4. FTIR spectra were recorded in the transmittance mode with $4 \mathrm{~cm}^{-1}$ resolution. The characteristic spectra of the PLGA polymer showed the $-\mathrm{CH},-\mathrm{CH} 2,-\mathrm{CH} 3$ stretching at $2850-3000 \mathrm{~cm}^{-1}, \mathrm{C}-\mathrm{O}$ stretching at $1050-1250 \mathrm{~cm}^{-1}$, carbonyl $-\mathrm{C}=\mathrm{O}$ stretching at $1700-1800 \mathrm{~cm}^{-1}$. In the encapsulated nanoparticles, some peaks were slightly moved to a lower wavelength and some peaks were not observed that are present in individual components.

\section{Encapsulation efficiency}

The encapsulation and efficiency of the particle was around $62 \%$ and indicating that some portion of TQ was portioned out of PLGA during the emulsification process. In earlier reports, 63\% entrapment efficiency was reported for TQchitosan nanoparticles and $90 \%$ for TQ-liposome nanoparticles (Alam et al., 2012; Odeh et al., 2012). 97.5\% efficiency was reported in PLGA in combination with polyethylene glycol (PEG)-5000 as a using stabilizer (Ravindiral et al., 2010). The lower encapsulation efficiency of TQ in the present study may be due to the PVA stabiliser used. PVA is one of the widely used stabilising agent and therefore preferably used in the present study over other agents like didodecyl dimethyl ammonium bromide (DMAB), and Sodium dodecyl sulphate (Mora-Huertas et al., 2010).

\section{In-vitro release kinetics}

The release of thymoquinone was studied as a function of $\mathrm{pH}$ over the period of 100 hours. $0.1 \mathrm{M} \mathrm{HCl}$ and $0.1 \mathrm{M}$ PBS was used as simulating the condition of gastric juice and intestinal juice conditions, respectively. It was observed (figure 5) that there was a burst release of TQ in initial 10 hours period with $25 \%$ release of TQ from nanoparticles and this may be due to rapid dispersion of TQ present in the surface of the PLGA. Thereafter, a sustained release of TQ was observed up to $75 \%$ for intestinal condition and $54 \%$ for the gastric at the end of the 7 days. The study indicate that the release rate was higher in acidic $\mathrm{pH}$ that the neutral $\mathrm{pH}$. This kind of biphasic release is in consistent with other previous studies (Zolnik et al., 2006; Tsai et al., 2011).

\section{In vitro anti-oxidant activity}

TQ has antioxidant activity and therefore the effect of PLGA on the TQ nanoparticle was studied in DPPH radical scavenging assay. DPPH assay is a stable free radical method and it is an easy, rapid and sensitive way to survey the antioxidant activity of specific compounds. The principle of DPPH method is based on the reduction of $\mathrm{DPPH}$ in the presence of a hydrogen donating antioxidant due to the formation of diphenyl picryl hydrozine. Sample compounds reduce the colour of DPPH due to the power of hydrogen donating ability. DPPH radical scavenging power of TQ nanoparticles were at $71.23 \%$ (figure 6) of inhibition at $1000 \mu \mathrm{g} / \mathrm{ml}$ concentration and for BHA $(84.14 \%$ of inhibition). Discolouration of violet DPPH to yellow clearly demonstrated the effect of NPs as an antioxidant. In a previous study by Mathew et al., (2012), reported that the PLGA coated curcumin nanoparticles had conserved antioxidant property.

\section{Effect of temperature on nanoparticles}

Figure 7 shows the effect of heat treatment at $60^{\circ}, 80^{\circ}$ and $100^{\circ} \mathrm{C}$ on size and zeta potential of TQ-PLGA Nanoparticle. It was observed that the size was rapidly reduced in the first $5 \mathrm{~min}$ at all the temperatures and consequently there was a slight change on the zeta potential. Heat treatment therefore affected the surface of the particles could have resulted in lowering of zeta potential. Overall, the results showed that PLGA nanoparticles were maintained within a regular size over the period of $15 \mathrm{~min}$ at various temperatures indicating physical properties of the nanoparticles were slightly affected by heat treatments.

\section{Antibacterial property by well diffusion method}

Thymoquinone possesses antimicrobial activity against many pathogens (Kouidhi et al., 2011). We tested this property in a PLGA coated TQ by agar diffusion method (figure 8). Results obtained from study shows that TQ nanoparticles possess antibacterial property against $E$. coli, Staphylococcus aureus and Salmonella typhi with inhibition zone diameter (IZD) of 6, 7 and $7 \mathrm{~mm}$, respectively.

\section{CONCLUSION}

In the present study, TQ loaded PLGA nanoparticles were synthesised with a relative homogeneity in size distribution of particles with less than $<200 \mathrm{~nm}$ size. Encapsulation of TQ did not result in destruction of its antioxidant and antibacterial activity. TQ- PLGA nanoparticles with sustained and controlled release properties can therefore be one of the promising methods of delivery system. As a therapeutic agent, the encapsulated TQ can be used in fortifying various food matrixes. 


\section{REFERENCES}

Abdel-Wahab, W.M. (2013). Protective effect of thymoquinone on sodium fluoride-induced hepatotoxicity and oxidative stress in rats. The Journal of Basic and Applied Zoology, (In Press). [DOI]

Ahsan, F., Rivas, I.P., Khan, M.A. and Torres Su'arez, A.I. (2002). "Targeting to macrophages: role of physicochemical properties of particulate carriers-liposomes and microspheres-on the phagocytosis by macrophages." Journal of Controlled Release, 79, 1-3, 29-40. [DOI]

Alam, S., Khan, ZI., Mustafa, G., Kumar, M., Islam, F., Bhatnagar, A. and Ahmad, F.J. (2012). Development and evaluation of thymoquinoneencapsulated chitosan nanoparticles for nose-to-brain targeting: a pharmacoscintigraphic study. International Journal of Nanomedicine, 7, 5705-18. [DOI]

Alhebshi, A.H., Gotoh. M. and Suzuki, I. (2013). Thymoquinone protects cultured rat primary neurons against amyloid $\beta$-induced neurotoxicity. Biochemical and Biophysical Research Communications. 19, 433(4), 362-7. [DOI]

Al-Majed, A., Al-Omar, F.A. and Nagi, M.N. (2006). Neuroprotective effects of thymoquinone against transient forebrain ischemia in the rat hippocampus. European Journal of Pharmacology, 543, 1-3, 14, 40-47. [DOI]

Arslan, S.O., Gelir, E., Armutcu, F., Coskun, O., Gurel, A., Sayan, H. and Celik, I.L. (2005). The protective effect of thymoquinone on ethanolinduced acute gastric damage in the rat. Nutrition Research, 25, 673680. [DOI]

Bhattacharyya, S.S., Paul, S. and Khuda-Bukhsh, A.R. (2010). Encapsulated plant extract (Gelsemium sempervirens) poly (lactide-co-glycolide) nanoparticles enhance cellular uptake and increase bioactivity in vitro Experimental Biology and Medicine, 235, 678-688. [DOI]

Braca, A., Tommasi, N.D., Bari, L.D., Pizza, C., Politi, M. and Morell,i I. (2001). Antioxidant principles from Bauhinia tetrapotensis. Journal of Natural Products, 64, 892-895. [DOI]

Das, J., Das, S., Samadder, A., Bhadra, K. and Khuda-Bukhsh, A.R. (2012). Poly (lactide-co-glycolide) encapsulated extract of Phytolacca decandrademonstrates better intervention against induced lung adenocarcinoma in mice and on A549 cells. European Journal of Pharmaceutical Sciences, 47, (2), 313-324. [DOI]

Derakhshandeh, K., Hosseinalizadeh, A. and Nikmohammadi M. (2011) The effects of PLGA microparticles on intestinal absorption of $\mathrm{p}-$ glycoprotein substrate using the everted rat intestinal sac model. Archives of Pharmacal Research, 34, (11), 1989-1997. [DOI]

El-Mahmoudy, A., Matsuyama, H., Borgan, M.A., Shimizu, Y., El-Sayed, M.G., Minamoto, N. and Takewaki T. (2002). thymoquinone suppresses expression of inducible nitric oxide synthase in rat macrophages International Immunopharmacology, 2, 11, 1603-1611. [DOI]

Gali-Muhtasib, H., Roessner, A. and Chneider-Stock, R. (2006). Thymoquinone : A promising anti-cancer drug from natural sources. The International Journal of Biochemistry and Cell Biology, 38, 8, 1249-1253. [DOI]

Ghosheh, O.A., Houdi, A.A. and Crooks. (1999). High performance liquid chromatographic analysis of the pharmacologically active quinines and related compounds in the oil of the black seed (Nigella sativa L). Journal of Pharmaceutical and Biomedical analysis, 19, 757-762. [DOI]

Harzallah, H.J., Kouidhi, B., Flamini, G., Bakhrouf, A. and Mahjoub, T. (2011).Chemical composition, antimicrobial potential against cariogenic bacteria and cytotoxic activity of Tunisian Nigella sativa essential oil and thymoquinone. Food Chemistry, 129, 4, 15, 1469-1474. [DOI]

Jang, K. and Lee, H.G. (2008). Stability of chitosan nanoparticles for Lascorbic acids during heat treatment in aqueous solution. Journal of agricultural and food chemistry, 56, 1936-1941. [DOI]

Kouidhi, B., Zmantar, T., Jrah, H., Souiden, Y., Chaieb, K., Mahdouani, K. and Bakhrouf, A. (2011). Antibacterial and resistance-modifying activities of thymoquinone against oral pathogens. Annal of Clinical Microbiology and Antimicrobials, 10, 29, 1-7. [DOI]

Kumari, A., Yadav, S.K., Pakade, Y.B., Kumar, V., Singh, B., Chaudhary, A. and Yadav, S.C. (2011). Nanoencapsulation and characterization of Albizia chinensis isolated antioxidant quercitrin on PLA nanoparticles. Colloids and Surfaces B: Biointerfaces, 82(1), 224-232. [DOI]

Ma, Y., Zhao, X., Li, J. and Shen, Q. (2012). The comparison of different daidzein-PLGA nanoparticles in increasing its oral bioavailability. International Journal of Nanomedicine, 7, 559-70. PMid:22346351 PMCid:PMC3277436

Magdy, M.A., Hanan, El-A. and Nabila, el-M. (2012). Thymoquinone: Novel gastroprotective mechanisms. European Journal Pharmacology. 15, 697(1-3), 126-31. [DOI]

Mansour, M.A., Nagi, M.N., El-Khatib, A.S. and Al-Bekairi, A.M. (2002) Effects of thymoquinone on antioxidant enzyme activities, lipid peroxidation and DT-diaphorase in different tissues of mice: a possible mechanism of action. Cell Biochemistry and Function, 20(2), 143-51. [DOI]

Mathew, A., Fukuda, T., Nagaoka, Y., Hasumura, T., Morimoto, H., Yoshida, Y., Maekawa, T., Kizhikkilot., Venugopal, K. and Kumar, D.S (2012). Curcumin Loaded-PLGA Nanoparticles Conjugated with Tet-1 Peptide for Potential Use in Alzheimer's Disease. PLoS ONE. www.plosone.org. [DOI]

Merlin, J.P.J., Prasad, N.R., Shibli, S.M.A. Sebeela, M. (2012). Ferulic acid loaded Poly-d,l-lactide-co-glycolide nanoparticles: Systematic study of particle size, drug encapsulation efficiency and anticancer effect in nonsmall cell lung carcinoma cell line in vitro. Biomedicine \& Preventive Nutrition, 2, (1), 69-76. [DOI]

Mora-Huertas, C.E., Fessi, H. and Elaissari, A. (2010). Polymer-based nanocapsules for drug delivery. International Journal of Pharmaceutics. 29;385(1-2):113-42. [DOI]

Odeh F, Ismail SI, Abu-Dahab R, Mahmoud IS, Al Bawab A. (2012). Thymoquinone in liposomes: a study of loading efficiency and biological activity towards breast cancer. Drug Delivery, 19 (8), 371-7. [DOI]

Okeke, M.I., Iroegbu, C.U., Eze, E.N., Okoli, A.S. and Esimone, C.O. (2001) Evaluation of extracts of the root of Landolphia owerrience for antibacterial activity. Journal of Ethnopharmacology, 78 (2-3), 119-27. [DOI]

Pari, L. and Sankaranarayanan, C. (2009).Beneficial effects of thymoquinone on hepatic key enzymes in streptozotocin-nicotinamide induced diabetic rats. Life Sciences, 85, 23-26, 830-834. [DOI]

Qi, L., Xu, Z., Jiang, X., Hu, C. and Zou, X. (2004). "Preparation and antibacterial activity of chitosan nanoparticles," Carbohydrate Research, 339, 16, 2693-2700. [DOI]

Ravindran, J., Nair, H.B., Sung, B., Prasad, S., Tekmal, R.R. and Aggarwal, B.B. (2010). Thymoquinone poly (lactide-co-glycolide) nanoparticles exhibit enhanced anti-proliferative, anti-inflammatory, and chemosensitization Potential. Biochemical Pharmacology, 79(11), 1640-1647. [DOI]

Samadder, A., Das, S., Das, J., Paul, A. and Khuda-Bukhsh, A.R. (2012) Ameliorative Effects of Syzygium jambolanum extract and its Poly (lactic-co-glycolic) Acid Nano-encapsulated Form on Arsenic-induced Hyperglycemic Stress: A Multi-parametric Evaluation. Journal of Acupuncture and Meridian Studies, 5, 310-318. [DOI]

Savic, R., Luo, L., Eisenberg, A. and Maysinger, D. (2003). Micellar nanocontainers distribute to defined cytoplasmic organelles. Science, 300, 615-18. [DOI]

Semete, B., Booysen, L., Lemmer, Y., Kalombo, L., Katata, L., Verschoor, J. and Swai., H.S. (2010). In vivo evaluation of the biodistribution and safety of PLGA nanoparticles as drug delivery systems. Nanomedicine: Nanotechnology, Biology and Medicine. 6, 662-671. [DOI]

Srivastava, A.K., Bhatnagar, P., Singh, M., Mishra, S., Kumar, P., Shukla, Y and Gupta, K.C. (2013). Synthesis of PLGA nanoparticles of tea polyphenols and their strong in vivo protective effect against chemically induced DNA damage. International Journal of Nanomedicine, 8, 1451-62. PMid:23717041 PMCid:PMC3663489

Tsai, Y.M., Chien, C.F., Lin, L.C. and Tsai, T.H. (2011). Curcumin and its nano-formulation: the kinetics of tissue distribution and blood-brain barrier penetration. International Journal of Pharmaceutics. 15, 416 (1), 331-8. [DOI]

Umar, S., Zargan, J., Umar, K., Ahmad, S., Katiyar, C.K. and Khan, H.A. (2012). Modulation of the oxidative stress and inflammatory cytokine response by thymoquinone in the collagen induced arthritis in Wistar rats. Chemico- Biological Interactions, 15, 197(1), 40-6. [DOI]

Ven, H.V.D., Vermeersch, M., Matheeussen, A., Vandervoort, J., Weyenberg, W., Apers, S., Cos, P. L. and Ludwig, M.A.L. (2011). PLGA nanoparticles loaded with the antileishmanial saponin $\beta$-aescin: Factor influence study and in vitro efficacy evaluation. International Journal of Pharmaceutics, 420, (1), 122-132. [DOI]

Woo, C.C., Kumar, A.P., Sethi, G. and Tan, K.H.B. (2012). Thymoquinone Potential cure for inflammatory disorders and cancer. Biochemical Pharmacology, 83, 4, 15, 443-451. [DOI]

Xie, X., Tao, Q., Zou, Y., Zhang, F., Guo, M., Wang, Y., Wang, H., Zhou, Q. and Yu, S. (2011). PLGA nanoparticles improve the oral bioavailability of curcumin in rats: characterizations and mechanisms. J Agric Food Chem. 14; 59(17): Pages 9280-9. [DOI]

Yallapu, M.M., Gupta, B.K., Jaggi, M. and Chauhan, S.C. (2010). Fabrication of curcumin encapsulated PLGA nanoparticles for improved therapeutic effects in metastatic cancer cells. Journal of Colloid and Interface Science, 351, 19-29. [DOI]

Zolnik, B.S., Leary, P. E. and Burgess, D.J. (2006). Elevated temperature acceleratedrelease testing of PLGA microspheres. Journal of Controlled Release, 112, 293-300. [DOI] 